NASATM-, 207564

\title{
WORST-CASE FLUTTER MARGINS FROM F/A-18 AIRCRAFT AEROELASTIC DATA
}

\author{
Rick Lind ${ }^{1} \quad$ Marty Brenner ${ }^{2}$ \\ NASA - Dryden Flight Research Center
}

\begin{abstract}
An approach for computing worst-case flutter margins has been formulated in a robust stability framework. Uncertainty operators are included with a linear model to describe modeling errors and flight variations. The structured singular value, $\mu$, computes a stability margin which directly accounts for these uncertainties. This approach introduces a new method of computing flutter margins and an associated new parameter for describing these margins. The $\mu$ margins are robust margins which indicate worst-case stability estimates with respect to the defined uncertainty. Worst-case flutter margins are computed for the F/A-18 SRA using uncertainty sets generated by flight data analysis. The robust margins demonstrate flight conditions for flutter may lie closer to the flight envelope than previously estimated by $p-k$ analysis.
\end{abstract}

\section{Introduction}

Aeroelastic flutter is a potentially destructive instability resulting from an interaction between aerodynamic, inertial and structural forces [4]. Design of a new aircraft, or even a configuration change of a current aircraft, requires study of the aeroelastic stability before a safe flight envelope can be determined. The aeroelastic community has identified several areas of research that are essential for developing an accurate flutter test program [6]. These areas focus on the dramatic time and cost associated with safely expanding the flight envelope to ensure no aeroelastic instabilities are encountered.

An important research topic for aeroelasticity engineers is the development of more confident futter or instability margins. Experimental methods of determining lutter usually consist of approximating modal

\footnotetext{
'NRC PoutDoctoral Research Fellow, NASA, Dryden Flight Research Ceater, MS 4840 D/RC, Edwards, CA 93523-0273, 805.258.3075, lindOxrd.dfre.nasa.gov, Member ALAA

${ }^{2}$ Research Engineer, gonzo Oxrd.dfrc.nasa.gov

- AIAA Structurea, Structural Dynamics and Materials Conference, ALAA-97-1266, Oriando FL, April 1997
}

damping from flight data [11]. These methods are unreliable due to the often sudden onset of flutter which may not be accurately indicated by an approximate damping value.

Several analytical methods are developed to determine the conditions for aeroelastic instability. A traditional method, known as the $p-k$ method, utilizes a structural model coupled with equations for the unsteady aerodynamics [12]. This method is based on a finite element model of the aircraft and does not directly consider flight data from the physical aircraft. A parameter estimation algorithm is developed that utilizes flight data to formulate elements of a state-space model [19]. This method suffers from poor excitation and data measurements that may lead to inaccurate modal parameters.

A novel approach to computing flutter instability boundaries has been developed that utilizes a theoretical model while directly accounting for variations with flight data [14]. The aeroelastic stability problem is formulated in a framework suitable for well developed robust stability theory. Flight data is analyzed to describe'a set of uncertainty operators that account for variations between the theoretical model and the physical aircraft. A robust stability measure known as the structured singular value, $\mu$, is used to compute flutter boundaries that are robust to these variations [2]. In this sense, a worst-case flutter boundary is computed that directly accounts for flight data.

This paper computes robust, or worst-case, flutter margins for the F/A-18 Systems Research Aircraft, SRA, being flown at NASA Dryden Flight Research Center. The SRA is a two-seat configuration fighter with production engines. Recent flutter testing was initiated due to a structural modification to the left wing. Internal fittings were replaced with larger and heavier ones to accommodate flight testing advanced aileron concepts. The flight data presented in this paper was generated using the new internal fitings but with a standard aileron. A wingtip excitation system for generating aeroelastic light data is shown in Figure 1. 


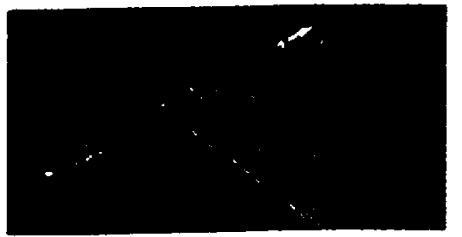

Figure 1: F/A-18 Wing with DEI Exciter

The lutter results in this paper represent a significant improvement to accepted flutter results for the F/A18 SRA computed using the traditional $p-k$ method. Nominal Butter margins computed using the $\mu$ method but ignoring all uncertainty operators are shown to match closely with the $p-k$ method lutter margins. This result lends validity to the $\mu$ method as an accurate indicator of Butter instability. Directly accounting for modeling uncertainty and light data variations in the $\mu$ based futter analysis generates robust flutter margins which are more conservative than the nominal margins.

These robust flutter margins are generated with a great deal more confidence than the nominal flutter margins. Flight data from the actual aircraft is analyzed to generate realistic uncertainty operators that ensure the family of plant models covers the true aircraft dynamics. Robust stability theory guarantees the robust flutter margins are worst-case margins with respect to the indicated amount of modeling uncertainty. This procedure may greatly reduce the time and cost associated with experimental flight envelope testing since the instability limits may be more accurately and confidently identified. Additionally, the uncertainty levels in the theoretical model may be determined using flight data from a safe flight condition without requiring the aircraft to approach a flutter instability point.

\section{Robust Stability and $\mu$}

Any aeroelastic model is an approximate representation of the aircraft dynamics. Inaccuracies in the model, such as errors in coefficients and unmodeled dynamics, must be considered in the stability analysis and control synthesis procedures. Uncertainty operators are included in the system model to account for these inaccuracies in the robust stability framework.

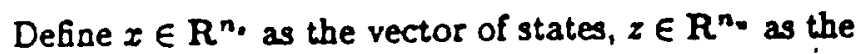

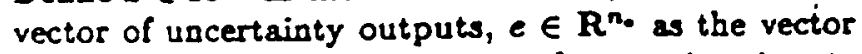
of errors, $w \in R^{n}$ as the vector of uncertainty inputs and $d \in \mathbf{R}^{n d}$ as the vector of disturbances. The statespace description of a linear time-invariant plant can be represented as

$$
\left[\begin{array}{c}
\dot{x} \\
z \\
e
\end{array}\right]=\left[\begin{array}{ccc}
A & B_{1} & B_{2} \\
C_{1} & E_{11} & E_{12} \\
C_{2} & E_{21} & E_{32}
\end{array}\right]\left[\begin{array}{l}
x \\
w \\
d
\end{array}\right]
$$

where $A \in \mathbf{R}^{n_{0} \times n_{0}}, B_{1} \in \mathrm{R}^{n_{0} \times n_{-}}, B_{2} \in \mathbf{R}^{n_{0} \times n_{4}}, C_{1} \in$

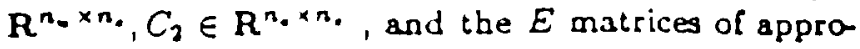
priate dimensions.

Define $P(s)$ as the Laplace transform of this system. The system with plant and uncertainty operators is represented as a Linear Fractional Transformation (LFT) of plant, $P$, and uncertainty operator, $\Delta$, in Figure 2.

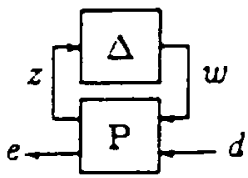

Figure 2: Robust Stability Framework

The uncertainty operator is allowed to lie within a norm bounded set. This leads to the consideration of a family of plant models. Weighting matrices are usually included to restrict the uncertainty norm bound to unity.

$$
\Delta=\left\{\Delta:\|\Delta\|_{\infty} \leq 1\right\}
$$

Robust stability considers stability of the system over the entire range of uncertainty. The issue of robust stability for LFT systems is associated with wellposedness to guarantee that all internal signals are finite and bounded. The small gain theorem is used to define robust stability for LFT systems $[2,18]$ :

Complex systems can have several types of uncertainty operators. Treating these types separately leads to structured uncertainty. It is well known robustness measured using the small gain theorem can be overly conservative for systems with structured uncertainty.

Define the structured singular value, $\mu$.

$$
\mu(P)=\frac{1}{\min \{\bar{\sigma}(\Delta): \Delta \in \Delta, \operatorname{det}(I-P \Delta)=0\}}
$$

$\mu$ is an exact measure of robustness for systems with structured uncertainty. The inverse of $\mu$ can be interpreted as a measure of the smallest destabilizing perturbation. The system is guaranteed to be robustly stable for all uncertainty operators bounded by the smallest destabilizing value.

Theorem 0.1 Given stable operator $P$, the system in Figure 2 is well-posed and stable for all $\Delta \in \Delta$ with $\|\Delta\|_{\infty}<1$ if and only if $\mu(P)<1$.

Unfortunately, $\beta$ is difficult to compute. Upper and lower bounds for $\mu$ have been derived which utilize two sets of structured scaling matrices [7]. These scaling matrices are similar in structure to the uncertainty block structure and commute with the uncertainty elements. An upper bound can be written as a linear matrix inequality (LMII) by considering a maximum eigenvalue value condition utilizing the structured scaling matrices [2]. 


\section{Worst-Case Flutter Method}

A worst-case method of computing flutter margins utiLizes $\mu$-analysis for evaluating system stability. A linear system is formulated with associated uncertainty operators.

Consider the generalized equation of motion for the structural response of the aircraft [10].

$$
M \ddot{\eta}+C \dot{\eta}+K \eta+\bar{q} Q(s) \eta=0
$$

For a system with $n$ modes, define $M \in \mathrm{R}^{n \times n}$ as the mass matrix, $C \in \mathbf{R}^{n \times n}$ as the damping matrix and $K \in \mathbf{R}^{n \times n}$ as the stiffness matrix. $\bar{q} \in \mathbf{R}$ is a scalar representing the dynamic pressure and $Q(s) \in C^{n \times n}$ is the matrix of unsteady aerodynamic forces.

The unsteady aerodynamic forces are fit to a standard finite-dimensional state-space system. This form can be shown to encompass the traditional forms of Roger and Karpel that include lag terms for the transient aerodynamics [14].

$$
Q(s)=\left[\begin{array}{ll}
A_{Q} & B_{Q} \\
C_{Q} & D_{Q}
\end{array}\right]=D_{Q}+C_{Q}\left(s I-A_{Q}\right)^{-1} B_{Q}
$$

Given the number of generalized states, $n$, and aerodynamic states, $n_{Q}$, define $A_{Q} \in \mathbf{R}^{n_{Q} \times n_{Q}}$, $B_{Q} \in \mathbf{R}^{n_{Q} \times n}, C_{Q} \in \mathbf{R}^{n \times n_{Q}}$ and $D_{Q} \in \mathbf{R}^{n \times n}$ as state-space elements approximating $Q(s)$.

The method should compute a $\mu$ value which relates an unstable fight conditions. This is accomplished by introducing an uncertainty operator to consider a range of fight conditions. Dynamic pressure is treated as an unknown quantity for worst-case flutter analysis.

Consider an additive perturbation, $\delta_{\bar{q}} \in \mathbf{R}$, on the nominal dynamic pressure, $\bar{q}_{\text {nom }}$.

$$
\bar{q}=\bar{q}_{\text {nom }}+\delta_{\bar{q}}
$$

Two signals, $z$ and $w$, are introduced into the formulation to represent uncertainty input and output. The uncertainty output is formulated from system states.

$$
z=M^{-1} D_{Q} \eta+M^{-1} C_{Q} x
$$

$w$ is related to $z$ by the dynamic pressure perturbation.

$$
w=\delta_{\bar{q}} z
$$

The state-space seroelastic model is formulated with the additional signals to account for the parameterization of the dynamic pressure uncertainty. Formulate the plant, $P(s)$, using state vector $[\eta ; \dot{\eta} ; x]$ such that $z=P(s) w$. Define $\hat{M}=-M^{-1}$.

$$
P=\left[\begin{array}{ccc|c}
0 & I & 0 & 0 \\
\dot{M}\left(K+\bar{q}_{\text {nom }} D_{Q}\right) & \hat{M} C & \bar{q}_{\text {nom }} \hat{M} C_{Q} & -I \\
B_{Q} & 0 & A_{Q} & 0 \\
\hline-\dot{M} D_{Q} & 0 & -\hat{M} C_{Q} & 0
\end{array}\right]
$$

The input to $P(s)$ is the uncertainty input, $w$, and the uncertainty output, $z$, is the output of $P(s)$. Defining additional signals for errors and disturbances allows $P(s)$ to be formulated in the robust stability framework of Figure 2 with $\delta_{\bar{q}}$ as the uncertainty operator.

Additional uncertainty operators are included to account for modeling errors between the theoretical system and the physical aircraft. They also allow the analysis to consider a range of aircraft dynamics that may change due to variations in parameters such as mass or variations in the aerodynamics such as small deflections in the aircraft surfaces.

Errors in elements of the state-space matrices are often represented by parametric uncertainty [3]. This uncertainty may be a real scalar parameter to reflect variation in physical parameters such as mass and dynamic pressure or real values such as modal frequency and damping.

Unmodeled dynamics and nonlinearities are often accounted for by including a complex uncertainty. The complex operator allows uncertainty to enter simultaneously in magnitude and phase of the signals. This dynamic uncertainty may be a scalar or a matrix reflecting unstructured uncertainty for a set of signals.

Experimental flight data can be used to generate uncertainty weightings. Transfer functions of the andytical model can be compared with experimental flight data transfer functions. Different size perturbations are allowed to affect specific system parameters to the degree that the resulting transfer functions cover the range of experimental tight data.

Model validation algorithms are used to verify that the amount of uncertainty in the linear model is sufficient to generate the flight data sets. This paper uses an atgorithm based on $\mu$-analysis of the linear system with frequency domain flight data $[14,13]$. The model validation condition is derived as a standard $\mu$ calculation. The $\mu$ value at each frequency relates the required size of perturbations at that frequency. This information is used to compute frequency varying weightings to scale the uncertainty set. The model validation procedure is repeated until a small amount of uncertainty is defined that still validates the model but reduces the conservatism in the resulting flutter analysis.

Robust flutter margins are computed using $\mu$-analysis on the linear system with the uncertainty operators. The flutter margin is found as the smallest destabilizing perturbation for the dynamic pressure uncertainty, $\delta_{\bar{q}}$, for the linear system with the given amount of modeling uncertainty. This margin is the worst-case flutter condition for the allowed range of aircraft dynamics. 


\section{Worst-Case Flutter Parameter}

The flutter computation method described in this paper uses $\mu$ as the worst-case lutter parameter. There are several advantages to using $\mu$ as the flutter parameter. $\mu$ is a much more informative Qutter margin as compared to traditional parameters such as pole location and modal damping.

The conservatism introduced by considering the worstcase uncertainty perturbation can be interpreted as a measure of sensitivity. Robust $\mu$ values which are significantly different than the nominal flutter margins indicate the plant is highly sensitive to modeling errors and changes in fight condition. A small perturbation to the system can drastically alter the futter stability properties. Conversely, similarity between the robust and nominal flutter margins indicates the aircraft is not highly sensitive to small perturbations.

Robustness analysis determines not only the norm of the smallest destabilizing perturbation but also the direction. This information relates exact perturbations for which the system is particularly sensitive. $\mu$ can thus indicate the worst-case flutter mechanism which may naturally extend to indicate active and passive control strategies for flutter suppression.

Damping is only truly informative at the point of instability since stable damping at a given fight condition does not necessarily indicate an increase in dynamic pressure will be a stable flight condition. $\mu$ computes the smallest destabilizing perturbation which indicates the nearest flight conditions that will cause a flutter instability. In this respect, $\mu$ is a stability predictor while damping is merely a stability indicator.

These characteristics of $\mu$ make the worst-case flutter algorithm especially valuable for tight test programs. Aeroelastic fight data can be measured at a stable flight condition and used to evaluate uncertainty operators. The $\mu$ method, unlike damping estimation, does not require the aircraft to approach instability for accurate prediction. $\mu$ can be computed to update the stability margins with respect to the new uncertainty levels. The worst-case stability margin then indicates what light conditions may be safely considered.

Safe and efficient expansion of the flight envelope can be performed using an on-line implementation of the worst-case stability estimation algorithm. Computing $\mu$ does not introduce an excessive computational burden since each F/A-18 lutter margin presented in this paper was derived in less than 2 minutes using standard off-the-shelf hardware and software packages. On-line algorithms are currently being developed to demonstrate this procedure for a fight test [17].
F/A-18 Aeroelastic Data

Extensive Gight data from the F/A-18 SRA is used to generate uncertainty descriptions for an analytical aircraft model [16]. Over 30 lights were conducted in two sessions between September 1994 and February 1995 and between June 1995 and July 1995 at Dryden Flight Research Center. Each light performed maneuvers for different conditions throughout the light envelope. $A$ total of 260 different data sets are generated from various conditions throughout the flight envelope [5].

The aeroelastic fight data is generated using an external structural excitation system developed by Dynamic Engineering Incorporated (DEI). This DEI exciter is a modification of an excitation system used for F-16 XL Alutter research [20]. The system consists of a wingtip exciter, an avionics box mounted in the instrumentation bay, and a cockpit controller.

Aerodynamic forces are generated by the wingtip exciter. This exciter consists of a small fixed aerodynamic vane forward of a rotating slotted hollow cylinder. Rotating the cylinder varies the pressure distribution on the vane and results in a wingtip force changing at twice the cylinder rotation frequency. The magnitude of the resulting force is determined by the amount of opening in the slot. The F/A-18 aircraft with a left side wingtip exciter is shown in Figure 1.

The cockpit controller commands a frequency range, duration and magnitude for the wingtip excitation signal. Frequency varying excitation is generated by changing the cylinder rotation frequency with sine sweeps. Each wingtip exciter is allowed to act inphase, 0 degrees, or out-of-phase, 180 ddegrees, with each other. Ideally, the in-phase data excites the symmetric modes of the aircraft and the out-of-phase data excites the anti-symmetric modes.

Flight data sets are recorded by activating the exciter system at a given light condition. The aircraft attempts to remain at the flight condition throughout the series of sine sweeps desired by the controller. The sine sweeps were restricted within $3 \mathrm{~Hz}$ and $35 \mathrm{~Hz}$. Smaller ranges were sometimes used to concentrate on a specific set of mode responses. Multiple sets of either linear or logarithmic sweeps were used with the sweep frequency increasing or decreasing.

Aeroelastic flight data generated with the DEI exciter system is analyzed by generating transfer functions from the excitation force to the sensor measurements. These transfer functions are generated using standard Fourier transform algorithms. There are several inherent assumptions associated with Fourier analysis that are violated with the flight data. The assumptions 
of time-invariant stationary data composed of sums of infinite sinusoids is not met by this transient response data. The analysis presented in this paper is based on Fourier analysis, although current research investigates wavelet techniques to analyze the light data [5].

The excitation force is not directly measured but rather a strain gauge measurement is used to approximate this force. The strain gauge records a point response at the exciter vane root. This point response is considered representative of the distributed excitation force load over the entire wing surface. Vane root strain is assumed to be directly proportional to the vane airloads due to excitation [5].

Analysis of the recorded flight data indicates the DEI exciters did not operate entirely as expected. The exciters displayed erratic behavior at higher dynamic pressures due to binding in both the motor drive mechanism and rotating cylinders. At low dynamic pressures the system operated better but still displays some phase drift between the left and right cylinder rotations.

Further erratic behavior is demonstrated by comparing measurement signals due to excitation sine sweeps of increasing and decreasing frequency. Transfer functions from a symmetric excitation to the wingtip accelerometers clearly show different modes are excited by the direction of the sweep even though the flight conditions are identical and the data sets were recorded 30 seconds apart of each other [16].

\section{F/A-18 Nominal Model}

The generalized equations of motion are used to derive a linear, finite-dimensional state-space model of the aircraft. This model contains 14 symmetric structural modes, 14 antisymmetric structural modes and 6 rigid body dynamic modes. The control surfaces are not active and no control modes are included in the model.

A finite element model of the SRA is used to compute the modal characteristics of the aircraft. Frequencies of the dominant modes for flutter are presented in $\mathrm{Ta}$ ble 1. These modal frequencies are computed for the aircraft with no aerodynamics considered. The predicted fluiter results for this aircraft are computed from the finite element model using the $p-k$ method. A detailed explanation of the SRA futter analysis using traditional methods is given in Reference [21].

Values of the unsteady aerodynamic force matrix at distinct frequencies are computed for the finite element model using a computer package developed for NASA known as STARS [9]. This code solves the subsonic aerodynamic equations using the doublet lattice

\begin{tabular}{|c|c|c|}
\hline Mode & Symmetric & AntiSymmetric \\
\hline Wing $1^{\text {si }}$ Bending & 5.59 & 8.84 \\
\hline Fuselage $1^{\prime \prime}$ Bending & 9.30 & 8.15 \\
\hline Wing $1^{\prime \prime}$ Torsion & 13.98 & 14.85 \\
\hline Wing $2^{\text {nd }}$ Bending & 16.95 & 16.79 \\
\hline Wing Outer Torsion & 17.22 & - \\
\hline Fuselage $2^{\text {nd }}$ Bending & 19.81 & 18.62 \\
\hline Fuselage Torsion & - & 24.19 \\
\hline Wing $2^{\text {nd }}$ Torsion & 29.88 & 29.93 \\
\hline
\end{tabular}

Table 1: Modal Frequencies

method [8]. The supersonic forces are generated using a different approach known as the constant panel method [1].

The doublet lattice and constant panel methods are used to compute the frequency varying unsteady aerodynamic forces for several subsonic,transonic and supersonic Mach numbers. The Mach numbers, $M=$ $.8, .9, .95,1.1,1.2,1.4,1.6$, are available. The unsteady aerodynamic forces are computed as a function of reduced frequency, $k$.

$$
k=\omega \frac{\bar{c}}{2 V}
$$

The reduced frequency is a function of the tiue frequency, $\omega$, the true velocity, $V$, and $\bar{c}$ the mean aerodynamic chord. Aerodynamic forces generated for 10 reduced frequency points between $k=.0001$ and $k=4$ are sufficient for flutter margin computation for this aircraft.

The unsteady aerodynamic forces are fit to a finitedimensional state-space system. The system identifcation algorithm is a frequency domain curve fitting algorithm based on a least squares minimization. A separate system is identified for each column of the unsteady forces transfer function matrix. $4^{\text {th }}$ order state-space systems are used for each column of the symmetric forces and $2^{\text {nd }}$ order state-space systems are used for each column of the antisymmetric forces. These systems are combined to form a single multipleinput and multiple-output state-space model of the unsteady aerodynamics forces, previously designated $Q(s)$, with 56 states for the symmetric modes and 28 states for the antisymmetric modes.

The analytical aeroelastic model has inputs for symmetric and antisymmetric excitation forces. It is assumed the excitation force will be purely symmetric or antisymmetric. There are 6 sensor measurements generated by accelerometers at the fore and aft of each wingtip and on each aileron. 


\section{F/A-18 Uncertainty Description}

Noise and uncertainty operators are introduced to the linear aeroelastic model to account for variations between the analytical model and the actual aircraft. These operators are developed by physical reasoning of the modeling process and also using the flight data generated by the DEI excitation system [16].

Standard analysis of the linear model is used to determine the framework for how uncertainty operators enter the system. Two uncertainty operators and a single noise input are used to describe the modeling uncertainty in the linear aeroelastic model. The magnitude of each uncertainty operator and the noise level is determined both from physical reasoning of the model and analysis of the tight data.

An uncertainty operator, $\delta_{\text {mode }}$, is introduced to the modal elements of the state-space F/A-18 model. This parametric uncertainty allows variations in both the natural frequency and damping values for each mode. This uncertainty covers errors in the coefficients of the equations of motion and the corresponding state-space elements of the linear model. An example of such an error arises in considering the mass of the aircraft. The linear model uses a single mass value while in reality the mass varies considerably due to fuel consumption. Mass variations for a simple second order system affect the natural frequency, $\omega=\sqrt{k / m}$, and may be represented as parametric modal uncertainty. This modal uncertainty allows a worst-case flutter point to be computed that accounts for parametric changes in the aircraft such as those due to mass variations.

The second uncertainty operator, $\Delta_{i n}$, is a multiplicative uncertainty on the force input to the linear model. This uncertainty is used to cover nonlinearities and unmodeled dynamics. The linear model contains no dynamics above $40 \mathrm{~Hz}$ so the high frequency component of this operator will reflect this uncertainty. This operator is also used to model the excitation uncertainty due to the DEI exciter system. Analysis of the Aight data indicates the input excitation signals rarely had the desired magnitude and phase characteristics that they were designed to achieve. The low frequency component of the input uncertainty reflects the uncertainty associated with the excitation system used to generate the flight data.

A noise signal is included with the sensor measurements. Knowledge of the aircraft sensors is used to determine a level of $10 \%$ noise is possible in the measured light data. An additional noise may be included on the force input due to the excitation system but it is decided the input multiplicative uncertainty is sufficient to describe this noise.
The magnitude of the parametric modal uncertainty, $\delta_{\text {mode, }}$ is determined trom light data analysis. The linear model contains 14 modes for the symmetric response and 14 modes for the antisymmetric response of the aircraft. Unfortunately, the fight data does not indicate each of these is sufficiently excited to allow analysis and comparison with the theoretical model. Only the modes given in Table 1 are observed in the data. A linear model is formulated from a subset of the full model which contains only the experimentally observed modes. The modal parameters of this reduced order model are compared with the light data and uncertainty levels are determined.

Scalar uncertainty parameters, $\delta$, are used to affect the modal parameters. The state matrix of the linear model is formulated as a block diagonal matrix with a $2 \times 2$ block for each mode. The diagonal component of each block is the real part of the natural frequency and the off-diagonal elements are the imaginary parts such that the natural frequency, $\omega_{i}$, and the damping, $\zeta_{i}$, of the $i^{\text {th }}$ mode may be determined.

$$
A_{i}=\left[\begin{array}{cc}
r & i \\
-i & r
\end{array}\right] \quad \Leftrightarrow \quad \begin{gathered}
\omega_{i}=\sqrt{r^{2}+i^{2}} \\
\zeta_{i}=-r / \omega_{i}
\end{gathered}
$$

Scalar weightings, $w_{r}$ and $w_{i}$, are used to affect the amount of uncertainty in each matrix element. The amount of variation in the matrix elements, and correspondingly the amount of variation in the natural frequency and damping, are determined by the magnitude of these scalar weightings. Define $\bar{r}$ and $\bar{i}$ as the varying elements of the state matrix affected by the uncertainty $\delta$.

$$
\begin{aligned}
& \bar{r}=r\left(1 \pm w_{r} \delta\right) \\
& \bar{i}=i\left(1 \pm w_{i} \delta\right)
\end{aligned}
$$

Aeroelastic modes typically show low damping values caused by the real component being quite small as compared to the imaginary component. Since linear modeling techniques often identify the natural frequency better than the damping value, the weighting for the real component should be larger than that for the imaginary component.

The weightings are chosen using the observed modal parameters in the fight data. The natural frequencies show variations of $\pm 5 \%$ from the theoretical model while the uncertainty in the damping needs approximately $\pm 15 \%$ to validate all the fight data. The scalar weightings are chosen accordingly.

$$
\begin{aligned}
& w_{r}=.15 \\
& w_{i}=.05
\end{aligned}
$$

The Aight data is only able to determine uncertainty levels for the modal paramters of the experimentally observed modes. It is assumed the uncertainty levels in the unobserved modes should be consistent with 
these values. Parametric uncertainty is introduced for each modal block in the state matrix, affecting observed and unobserved modes, with the weighting values given above.

The block diagonal state matrix also contains some real valued scalar blocks. These scalar blocks appear as approximations to lag terms in the state-space identification of the unsteady aerodynamic forces. The identified system with these lag approximations does not accurately model the aerodynamic forces at all frequencies. Parametric uncertainty affects each of these lag terms with a weighting of $w_{\text {lag }}=.15$ that allows $15 \%$ variation.

The low frequency magnitude of the input multiplicative uncertainty is determined from the flight data. Levels of uncertainty are chosen that validate the flight data for a given amount of noise and parametric modal uncertainty. The high frequency component of input uncertainty is determined to reflect the unknown dynamics at high frequency for the linear model. The frequency varying transfer function for weighting the input uncertainty is given as $W_{\text {in }}$.

$$
W_{\text {in }}=5 \frac{s+100}{s+5000}
$$

The block diagram for the aeroelastic model with the uncertainty operators is given in Figure 3.

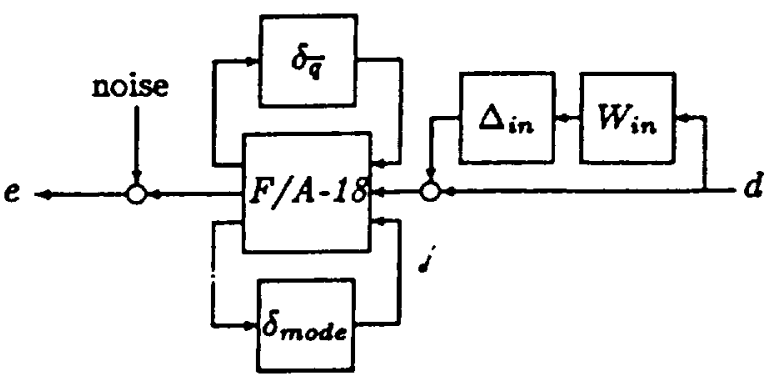

Figure 3: F/A-18 Uncertainty Block Diagram

Flight data used to validate this uncertainty structure covers a large range of flight points from the entire set of 260 flight maneuvers throughout the fight envelope.

Using a single uncertainty description over the entire tight envelope may be conservative. It is reasonable to assume the linear models are more accurate at subsonic and supersonic than at transonic. Additionally, the flight data from the DEI exciter system should be better at subsonic speeds than at supersonic. However, it simplifies the analysis process to consider a single set of uncertainty operators. This process is equivalent to formulating the worst-case uncertainty levels at the worst-case light condition and assuming that amount of uncertainty is possible for the remaining light conditions.

\section{F/A-18 Flutter Points}

Flutter margins are computed for a linear model with the associated modeling uncertainty structure using the $\mu$-analysis method [15]. Linear systems for symmetric and antisymmetric structural modes are separated for ease of analysis. These systems can easily be combined and analyzed as a single system; however, eigenvector analysis would be required to distinguish which critical flutter modes are symmetric and which are antisymmetric. Each system contains the same number of structural modes, 14, and the uncertainty descriptions are identical for each linear model.

The system given in Figure 3 contains three uncertainty blocks. The parametric uncertainty covering variations due to dynamic pressure, $\delta_{\bar{q}}$, is a scalar parameter repeated 14 times, once for each elastic mode. The parametric uncertainty block affecting the modal parameters, $\delta_{\text {modes, }}$ is a diagonal matrix with dimension equal to the number of states. Separate scalars along the diagonal represent uncertainty in each elastic mode, each mode in the aerodynamic force approximation, and each lag term. The uncertainty parameters for the modes are repeated two times while the parameters for the lag terms are single scalars. Define $\delta_{i}$ as the $i^{\text {th }}$ uncertainty parameter for the system ith $n_{m}$ modes and $n_{l}$ lag terms. The input multiplicative uncertainty block, $\Delta_{i n}$, is a scalar for this siffgle input plant model since we are analyzing symmetric excitation separately from antisymmetric excitation.

The parametric uncertainty parameters represent changes in elements of the state-space model. The variation of $\delta_{\bar{q}}$ between \pm 1 admits dynamic pressures between $0 \leq \bar{q} \leq 2 \bar{q}_{\text {nom }}$. Allowing the modal uncertainty parameters, $\delta_{1}, \ldots, \delta_{n_{m}}$ to vary between \pm 1 allows $5 \%$ variation in the imaginary part of the natural frequency and $15 \%$ in the real part. This corresponds to approximately $5 \%$ variation in the natural frequency and $15 \%$ in the damping value of each mode. These parameters are real quantities. The multiplicative input uncertainty contains magnitude and phase information and is treated as a complex linear timeinvariant uncertainty.

Nominal flutter boundaries are initially computed by ignoring the modal and input uncertainties. $\mu$ is computed only with respect to the parametric uncertainty allowing a range of dynamic pressures to be considered. Robust flutter boundaries are computed with respect to the structured uncertainty set, $\Delta$, described above using the structured singular value. Traditional flutter boundaries computed using the $p-k$ method are presented with the nominal and robust flutter boundaries computed with $\mu$ in Table 2 


\begin{tabular}{|c||c|c|c||c|c|c|}
\hline \hline \multicolumn{1}{|c||}{ Mach } & \multicolumn{3}{c||}{ symmetric } & \multicolumn{3}{c|}{ antisymmetric } \\
\cline { 2 - 7 } & $\bar{q}_{p-k}$ & $\bar{q}_{\text {nom }}$ & $\bar{q}_{\text {rob }}$ & $\bar{q}_{p-k}$ & $\bar{q}_{\text {nom }}$ & $\bar{q}_{\text {rob }}$ \\
\hline .8 & 3360 & 3168 & 2909 & 4600 & 4593 & 3648 \\
\hline .9 & 2700 & 2706 & 2575 & 3150 & 3057 & 2944 \\
\hline .95 & 2430 & 2388 & 2329 & 2600 & 2751 & 2572 \\
\hline 1.1 & 5400 & 5676 & 4120 & 5500 & 3265 & 2827 \\
\hline 1.2 & $\mathbf{2 4 6 9}$ & 2454 & 2327 & 2850 & 2893 & 2653 \\
\hline 1.4 & $\mathbf{3 5 2 8}$ & $\mathbf{3 4 3 2}$ & $\mathbf{3 0 3 4}$ & 4600 & $\mathbf{4 4 3 9}$ & $\mathbf{4 1 9 1}$ \\
\hline 1.6 & $\mathbf{4 4 7 0}$ & $\mathbf{4 4 8 7}$ & $\mathbf{3 9 9 6}$ & $\mathbf{5 7 0 0}$ & 5870 & $\mathbf{4 5 3 6}$ \\
\hline \hline
\end{tabular}

Table 2: Nominal and Robust Flutter Points

The nominal flutter dynamic pressures computed using the $\mu$ method can be directly compared with those computed using the traditional $p-k$ method [21]. Each of these flutter solutions are based on an analytical model with no consideration of modeling uncertainty.

The nominal flutter points for the symmetric modes match closely with the $p-k$ method throughout the fight envelope. The subsonic and supersonic cases show an especially good correlation with the $p-k$ flutter points. For each of these flight regions, the $\mu$-analysis flutter dynamic pressures are nearly identical, within $1 \%$, to the $p-k$ method flutter dynamic pressures. The transonic case at $M=1.1$, however, shows a slight difference between the two methods. The $\mu$ method computes a flutter point that is greater than the $p-k$ method. In each Mach regime; subsonic,supersonic or transonic, the nominal flutter points are within $5 \%$ for the two methods.

The antisymmetric modes show a similar relationship between the flutter margins computed with the $\mu$ and $p-k$ methods. The subsonic and supersonic flutter points are within $5 \%$ for the two methods, but there is a greater deviation at the transonic condition. $\mu$ computes a flutter margin at $M=1.1$ that is $40 \%$ lower than the $p-k$ method indicates.

The nominal flutter points for the $\mu$ and $p-k$ methods show the greatest difference for both the symmetric and antisymmetric modes at the transonic case. The aerodynamics at $M=1.1$ are more difficult to model accurately than at either subsonic or supersonic. Numerical sensitivity to representations of the unsteady aerodynamic foces causes differences in the nominal Qutter margins.

The robust flutter margins computed using the $\mu$ method have lower dynamic pressures than the nominal margin, which indicates the expected conservative nature of the robust computation. These new flutter points are worst-case values for the entire range of allowed uncertainty. The subsonic and supersonic flutter boundaries are not greatly affected by the uncertainty set. In each of these cases, the robust flutter point is within $10 \%$ of the nominal lutter point.
The flutter boundary at the transonic case, $M=1.1$, demonstrates significant sensitivity to the modeling uncertainty. The robust lutter dynamic pressures are approximately $70 \%$ of the nominal Alutter margins. This is explained by considering the rapid transition of critical Autter boundaries near this region. The critical lutter frequencies and the flutter dynamic pressure widely vary between Mach numbers slightly lower and bigher than transonic. The small amount of modeling uncertainty is enough to cause the worst-case flutter mechanism to shift and the plant assumes characteristics more consistent with a non-transonic regime.

The modal natural frequencies for the critical flutter modes are presented in Table 3 . The frequencies computed using the $p-k$ method and the $\mu$-analysis method are close throughout the flight envelope for both the symmetric and antisymmetric modes. Frequencies for the robust flutter solutions are slightly different than the nominal flutter frequencies due to the modeling uncertainty which allowed $5 \%$ variation in the modal natural frequencies.

\begin{tabular}{|c||c|c|c||c|c|c|}
\hline \multicolumn{1}{|c||}{ Mach } & \multicolumn{3}{c||}{ symmetric } & \multicolumn{3}{c|}{ antisymmetric } \\
\cline { 2 - 7 } & $\omega_{p-k}$ & $\omega_{\text {nom }}$ & $\omega_{\text {rob }}$ & $\omega_{p-k}$ & $\omega_{\text {nom }}$ & $\omega_{\text {rob }}$ \\
\hline .8 & 8.2 & 7.6 & 7.7 & 9.0 & 9.1 & 9.1 \\
\hline .9 & 7.4 & 7.3 & 7.3 & 9.2 & 9.1 & 9.2 \\
\hline .95 & 6.8 & 6.9 & 6.9 & 9.1 & 9.2 & 9.2 \\
\hline 1.1 & 12.1 & 13.2 & 13.0 & 28.6 & 28.0 & 28.3 \\
\hline 1.2 & 26.5 & 27.4 & 27.4 & 26.9 & 28.9 & 28.9 \\
\hline 1.4 & 28.1 & 28.1 & 28.1 & 30.4 & 31.7 & 31.8 \\
\hline 1.6 & 28.9 & 30.1 & 30.1 & 32.8 & 32.3 & 32.1 \\
\hline \hline
\end{tabular}

Table 3: Nominal and Robust Flutter Frequencies

Subcritical flutter margins computed with the $\mu$ and $p-k$ methods are presented in Table 4. Only nominal subcritical margins are detected with $\mu$ since the robust margins are always worst-case critical margins.

\begin{tabular}{|c||c|c||c|c|}
\hline \multicolumn{1}{|c||}{ Mach } & \multicolumn{2}{c||}{ symmetric } & \multicolumn{2}{c|}{ antisymmetric } \\
\cline { 2 - 5 } & $\bar{q}_{p-k}$ & $\bar{q}_{\text {nomingl }}$ & $\bar{q}_{p-k}$ & $\bar{q}_{\text {nominal }}$ \\
\hline \multirow{2}{*}{.9} & & & 4700 & 4583 \\
& & & 5300 & 5093 \\
\hline .95 & 7450 & 6919 & & \\
\hline 1.1 & & & 6050 & 6001 \\
\hline 1.2 & 5400 & 5003 & 8400 & 7943 \\
\hline 1.4 & 8970 & 8959 & & \\
\hline 1.6 & 8400 & 8843 & & \\
\hline
\end{tabular}

Table 4: Nominal and Robust Flutter Points - Subcritical $\mu$-analysis computes subcritical Autter margins within $10 \%$ of the $p-k$ method for both the symmetric and antisymmetric modes. The $\mu$ method is evea able to detect the subcritical Gutter hump mode occuring for antisymmetric excitation at $0.9 \mathrm{Mach}$ number. 


\section{Matched-Point Flutter Margins}

The dynamic pressures at which glutter occurs are converted into altitudes, commonly known as matchedpoint solutions, using standard atmospheric equations. These altitudes are plotted for the symmetric modes in Figure 4 and for the antisymmetric modes in Figure 5. The flight envelope of the F/A-18 is shown on these plots along with the required $15 \%$ flutter boundary for military aircraft.

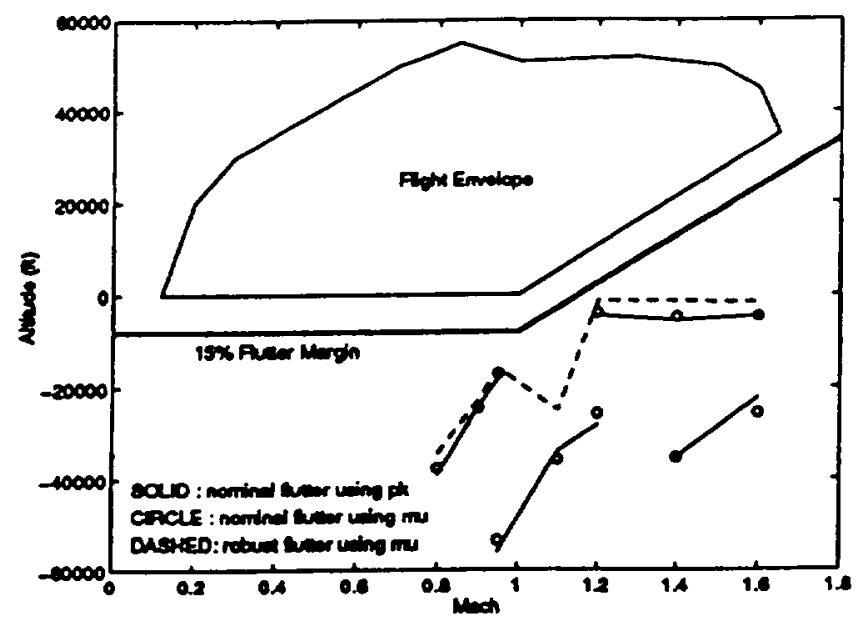

Figure 4: Nominal and Robust Flutter Points - Matched Point Solutions for Symmetric Modes

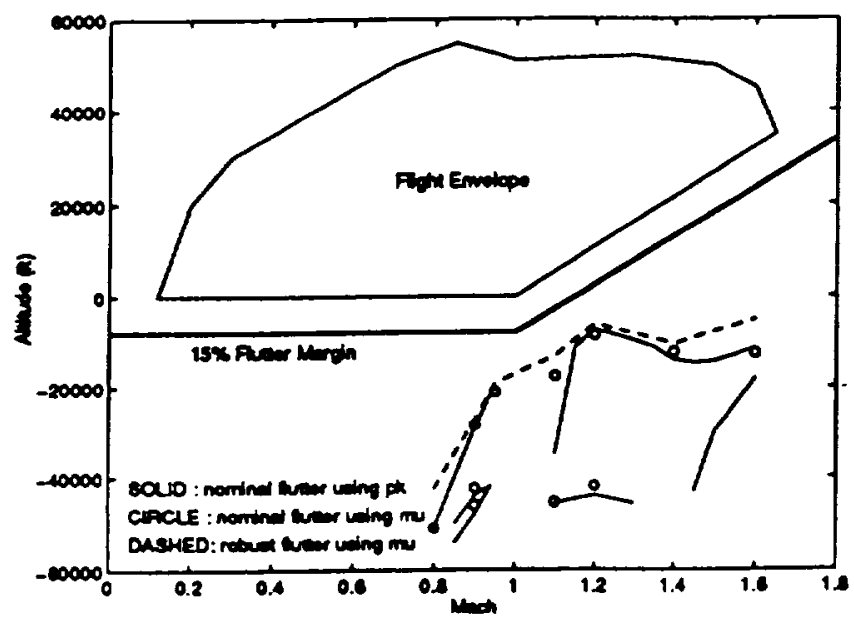

Figure 5: Nominal and Robust Flutter Points - Matched Point Solutions for AntiSymmetric Modes

Figures 4 and 5 use several short solid lines to indicate the $p-k$ futter solutions throughout the fight regime. Each of these short solid lines represents the futter points due to a specific mode. Flutter points for the symmetric modes given in Figure 4 show four solid lines indicating three different critical gutter modes for the considered range of Mach numbers along with a subcritical lutter mode occuring at supersonic Mach numbers. The antisymmetric modes show the onset of lutter from three different critical modes along with three subcritical flutter modes throughout the fight envelope in Figure 5. The frequencies of the critical Hutter modes can be found in Table 3.

The subsonic flutter altitudes for symmetric and antisymmetric modes demonstrate a similar characteristic. The nominal lutter boundary shows a significant variation from Mach number $M=.8$ to $M=.95$ caused by sensitivity to Mach number for the dynamics associated with the critical flutter mode. The robust futter boundary indicates the sensitivity of the plant to errors and the worst-case perturbation. The higher altitude for the nominal flutter boundary at Mach number $M=.81$ than for Mach number $M=.80$ is reflected in the large conservatism associated with the robust flutter boundary. Similarly, slight variation of Mach number near $M=.95$ is not expected to increase the nominal futter boundary so there is less conservatism associated with the robust flutter boundary.

An interesting trend is noticeable for the symmetric mode robust flutter points in Figure 4 at the supersonic Mach numbers. The flutter mechanism results from the same modes from $M=1.2$ to $M=1.6$ with some increase in frequency. Similarly the altitudes of the nominal Butter margins show little change for these Mach numbers. The aeroelastic dynamics associated with the critical flutter mode are relatively unaffected by the variation of Mach over this range and consequently each flutter boundary has the same sensitivity to modeling errors.

The robust flutter margins for the antisymmetric modes at supersonic Mach nuribers show a slightly different behavior than the symmetric mode flutter margins. The flutter mechanism is again caused by a single mode from $M=1.2$ to $M=1.6$ with similar frequency variation as symmetric. The robust flutter margins demonstrate a similar sensitivity to modeling errors at $M=1.2$ and $M=1.4$ but at $M=1.6 \mathrm{a}$ greater sensitivity is shown. The greater conservatism at $M=1.6$ may indicate impending transition in flutter mechanism from the subcritical mode at slightly higher Mach number.

The dark solid line on Figures 4 and 5 represents the required boundary for flutter points. All nominal and robust flutter points lie outside this region indicating the flight envelope should be safe from flutter instabilities. The robust flutter boundaries computed with $\mu$ indicate there is more danger of encountering flutter than was previously estimated with the $p-k$ method. In particular, the robust flutter margin for symmetric excitation at Mach $M=1.2$ lies considerably closer to the boundary than the $p-k$ method indicates. 


\section{Computational Analysis}

The $\mu$ analysis method of computing Autter margins presents significant analytical advantages due to the robustness of the resulting flutter margin, but it also has several computational advantages over the $p-k$ method. The $\mu$ algorithm requires a single linear aeroelastic plant model at a given Mach number to compute critical and subcritical lutter margins. An entire set of flutter margins may be easily generated using a standard engineering workstation in a few minutes using widely available software packages [2].

The $p-k$ method is an iterative procedure that requires finding a matched-point solution [21]. The aircraft is analyzed at a particular Mach number and air density. The airspeed for these conditions resulting in a flutter instability is computed. This airspeed, however, often does not correspond to the unique airspeed determined by that Mach number and air density for a standard atmosphere. Various air densities are used to compute flutter solutions and the corresponding air speeds are plotted. An example of an air speed plot for flutter is given in Figure 6.

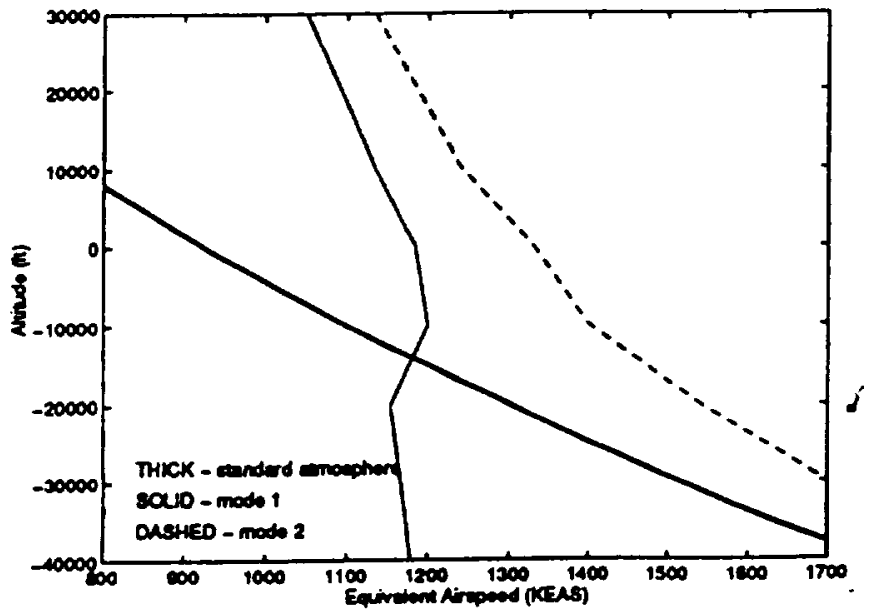

Figure 6: AntiSymmetric P.K Flutter Solutions for Mach $M=1.4$

The vertical lines in Figure 6 represent two antisymmetric modes that may lutter at Mach $M=1.4$. The $p-k$ method computes a flutter solution at the airspeed indicated where the modal line crosses the standard atmosphere curve. This flutter solution is difficult to compute from only a few air density computations. Typically several air densities are used to compute air speed flutter solutions and a line is extrapolated between the points to determine the matched-point solution at the standard atmosphere crossing point. The nonlinear behavior shown for mode 1 near the standard atmosphere crossing point indicates an accurate flutter boundary would be extremely hard to predict unless many solutions are computed near the true matchedpoint solution.

The $p-k$ method also may have difficulty predicting the subcritical lutter margins. The second mode in Figure 6 may or may not intersect the standard atmosphere curve. More computational analysis is required to determine the behavior of this mode at higher airspeeds. The $\mu$-analysis method accurately detects both the critical and subcritical flutter margins without requiring expensive iterations.

\section{Conclusion}

Nominal and robust Butter margins are computed for the F/A-18 SRA aircraft. Nominal flutter margins are computed using a $\mu$-analysis method and a traditional $p-k$ method. The similarity of these flutter margins demonstrates the $\mu$-analysis method is a valid tool for computing flutter instability points and is computationally advantageous. Extensive flight data is analyzed to develop a set of uncertainty operators for a linear model. Robust flutter margins are computed using $\mu$. The resulting flutter margins are worst-case values with respect to the modeling uncertainty. These margins are accepted with a great deal more confidence than previous flutter estimates by directly accounting for modeling uncertainty in the analysis process. The robust flutter margins indicate the desired flight envelope should be safe from aeroelastic flutter instabilities; however, the flutter margins may lie noticeably closer to the flight envelope than previously estimated.

This method replaces damping as a measure of tendency to instability from available Gight data. Since stability norms generally behave smoothly at instability boundaries, this method is recommended for preflight predictions and post-flight analysis with 8 minimum amount of fight time. Additionally, the robust Gutter stability framework extends naturally to robust futter control synthesis for aeroelastic control.

\section{Acknowledgments}

The authors wish to acknowledge the financial support of the Controls and Dynamics Branch of NASA at the Dryden Flight Research Center. The structural dynamics group, Larry Freudinger, Leonard Voelker and Dave Voracek, provided belpful comments and suggestions throughout this project. Analysis of the faite element model was assisted by Tim Doyle and Roger Truax. Dr. Lind is supported through the PostDoctoral Fellowship program of the National Research Council. 


\section{References}

[1] K. Appa, "Constant Pressure Panel Method for Supersonic Unsteady Airloads Analysis,", AIAA Journal of Aircroft, Vol. 24, No. 10, October 1987, pp. 696702.

[2] G. Balas, J. Doyle, K. Glover, A. Packard and R. Smith, " $\mu$-Analysis and Synthesis Toolbox - Users Guide,", MUSYN Inc and The MathWorks, Minneapolis, MN, 1991.

[3] G. Balas and P. Young, "Control Design for Variations in Structural Natural Frequencies," AIAA Journal of Guidance, Control and Dynamics, Vol. 18, No.2, March 1995, pp. 325-332.

[4] R. Bisplinghoff, A. Holt, and R. Halfman, Aeroelasticity, Addison Wesley Publishing, 1955.

[5] M. Brenner, R. Lind and D. Voracek, "Exciting Flutter Research with an F/A-18 Aircraft," 1997 AIAA Structures, Structural Dynamics and Materials Conference, Orlando FL, April 1997, Paper 97-1023.

[6] j. Cooper and T. Noll, "Technical Evaluation Report on the 1995 Specialists Meeting on Advanced Aeroservoelastic Testing and Data Analysis," Proceedings of the $80^{\text {th }}$ AGARD Structures and Materials Panel, AGARD-CP-566, Rotterdam, The Netherlands, May \&-10 1995, pp. T1-T10.

(7) M. Fan, A. Tits and J. Doyle, "Robustness in the Presence of Mixed Parametric Uncertainty and Unmodeled Dynamics," IEEE Trans. on Auto. Controh, Vol. 36, January 1991, pp. 25-38.

[8] J. Giesing, T. Kalman and W. Rodden, W., Subsonic Unsteady Aerodynamic for General Lattice Method, Part I - Vol I - Direct Application of the Nonplanar Doublet Lattice Method, AFFDL-TF-71-5, November 1971.

[9] K. Gupta, STARS - An Integrated General Purpose Finite Element Structural, Aeroelastic, and Aeroservoelastic Analysis Computer Program, NASA TM-101709, 1990.

[10] K. Gupta, M. Brenner and L. Voelker, Development of an Integrated Aeroservoelastic Analysis Program and Correlation with Test Dala, NASA Technical Paper TP-3120, May 1991.

[11] M. Kehoe, "A Historical Overview of Flight Flutter Testing," Proceedings of the $80^{\text {th }}$ AGARD Structures and Materials Panel, AGARD-CP-566, Rotterdam, The Netherlands, May 8-10 1995, pp. 1:11:15.

[12] HI. Hassig, "An Approximate True Damping Solution of the Flutter Equation by Determinant Iteration," AIAA Journal of Aircraft, Vol. 8, No. 11, November 1971, pp. 885-889.

[13] A. Kumar and G. Balas, "An Approach to Model Validation in the 4 Framework, "Proceedings of the
1994 American Controls Conference, Baltimore MD, June 1994, pp. 3021-3026.

[14] R. Lind and M. Brenner, "Robust Stability Estimation of Aeroelastic Systems using Flight Derived Uncertainty Models," submitted to AIAA Journal of Guidance, Control and Dynamics, June 1996.

[15] R. Lind and M. Brenner, "Robust Flutter Margins of an F/A-18 Aircraft from Aeroelastic Flight Data," submitted to AIAA Journal of Guidance, Control and Dynamics, July 1996.

[16] R. Lind and M. Brenner, "Incorporating Flight Data into a Robust Aeroelastic Model," submitted to AIAA Journal of Aircraft, September 1996.

[17] R. Lind and M. Brener, "A Worst-Case Approach for On-Line Flutter Prediction," to appers in 1997 CEAS International Forum on Aeroelasticity and Structural Dymamics, Rome Italy, June 1997.

[18] J. Maciejowski, Multivariable Feedback Design, Addison Wesley Publishers, England, 1989.

[19] E. Nissim and G. Gilyard, Method of Experimental Determination of Flutter Speed by Parometer Identification, NASA Technical Paper 2923, 1989.

[20] L. Vernon, In-flight Investigation of a Rotating Cylinder-Based Structural Excitation System for Flutter Testing, NASA Technical Memorandum 4512, June 1993.

[21] L. Voelker, F-18/SRA Flutter Analysis Resülts, preprint of NASA Technical Memorandum, 1995. 